\title{
On the observational behaviour of the highly polarized Type IIn supernova SN 2017hec
}

\author{
Brajesh Kumar ${ }^{\oplus},{ }^{1 \star}$ Chakali Eswaraiah, ${ }^{1,2 \star}$ Avinash Singh ${ }^{\oplus},{ }^{1,3 \star}$ D. K. Sahu, ${ }^{1}$ \\ G. C. Anupama, ${ }^{1}$ K. S. Kawabata, ${ }^{4}$ Masayuki Yamanaka, ${ }^{4,5}$ Ikki Otsubo, ${ }^{6}$ \\ S. B. Pandey, ${ }^{7}$ Tatsuya Nakaoka, ${ }^{6}$ Miho Kawabata, ${ }^{6}$ Amar Aryan ${ }^{7}$ and Hiroshi Akitaya ${ }^{4}$ \\ ${ }^{1}$ Indian Institute of Astrophysics, II Block, Koramangala, Bengaluru 560 034, India \\ ${ }^{2}$ National Astronomical Observatories, Chinese Academy of Sciences, Datun Road, Chaoyang District, Beijing 100101, P. R. China \\ ${ }^{3}$ Joint Astronomy Programme, Department of Physics, Indian Institute of Science, Bengaluru 560012 , India \\ ${ }^{4}$ Hiroshima Astrophysical Science Center, Hiroshima University, 1-3-1 Kagamiyama, Higashi-Hiroshima, Hiroshima 739-8526, Japan \\ ${ }^{5}$ Okayama Observatory, Graduate School of Science, Kyoto University, 3037-5 Honjo, Kamogata, Asakuchi, Okayama 719-0232, Japan \\ ${ }^{6}$ Department of Physical Science, Hiroshima University, Kagamiyama 1-3-1, Higashi-Hiroshima 739-8526, Japan \\ ${ }^{7}$ Aryabhatta Research Institute of Observational Sciences, Manora Peak, Nainital 263 001, India
}

Accepted 2019 July 3. Received 2019 June 6; in original form 2019 January 24

\begin{abstract}
We present the results based on photometric (Swift UVOT), broad-band polarimetric ( $V$ and $R$ bands) and optical spectroscopic observations of the Type IIn supernova (SN) 2017hcc. Our study is supplemented with spectropolarimetric data available in literature for this event. The post-peak light-curve evolution is slow $\left(\sim 0.2 \mathrm{mag} 100 \mathrm{~d}^{-1}\right.$ in $b$ band). The spectrum of $\sim+27 \mathrm{~d}$ shows a blue continuum with narrow emission lines, typical of a Type IIn SN. Archival polarization data along with the Gaia DR2 distances have been utilized to evaluate the interstellar polarization (ISP) towards the $\mathrm{SN}$ direction which is found to be $P_{\mathrm{ISP}}=0.17 \pm 0.02$ percent and $\theta_{\mathrm{ISP}}=140^{\circ} \pm 3^{\circ}$. To extract the intrinsic polarization of SN 2017hcc, both the observed and the literature polarization measurements were corrected for ISP. We noticed a significant decline of $\sim 3.5$ percent ( $V$ band) in the intrinsic level of polarization spanning a period of $\sim 2$ months. In contrast, the intrinsic polarization angles remain nearly constant at all epochs. Our study indicates a substantial variation in the degree of asymmetry in either the ejecta and/or the surrounding medium of SN 2017hcc. We also estimate a mass-loss rate of $\dot{M}=0.12 \mathrm{M}_{\odot} \mathrm{yr}^{-1}$ (for $v_{\mathrm{w}}=20 \mathrm{~km} \mathrm{~s}^{-1}$ ) which suggests that the progenitor of SN $2017 \mathrm{hcc}$ is most likely a luminous blue variable.
\end{abstract}

Key words: techniques: photometric - techniques: polarimetric - techniques: spectroscopicsupernovae: general - supernovae: individual: SN 2017hcc.

\section{INTRODUCTION}

Supernovae (SNe), of Type IIn are hydrogen rich core-collapse supernovae $\left(\mathrm{CCSNe}^{1}\right)$. They are primarily identified by their strong narrow- and intermediate-width emission components of $\mathrm{H}$, superimposed on a relatively smoother blue continuum (Schlegel 1990). These narrow emissions are believed to arise due to an interaction between the slow moving circumstellar material (CSM) and the faster moving SN ejecta (Chevalier \& Fransson 1994; Chugai \& Danziger 1994; Chugai 2001). Multiwavelength studies suggest

\footnotetext{
* E-mail: brajesh.kumar@iiap.res.in, brajesharies@gmail.com (BJ); swaraiahc@nao.cas.cn (CE); avinash21292@gmail.com (AS)

${ }^{1}$ These events characterize the explosion of massive stars $\left(>8 \mathrm{M}_{\odot}\right.$ ) (for a review, see Filippenko 1997).
}

that they cover a broad range of observational properties (Kiewe et al. 2012; Fox et al. 2013), pointing to the existence of subcategories of Type IIn SNe (see Taddia et al. 2013; Ofek et al. 2014; Smith 2017). The observed diversity depends upon various factors such as progenitor channels (Fransson et al. 2002; Gal-Yam et al. 2007; Dwarkadas 2011; Pastorello et al. 2013), mass-loss history (Smith 2014), explosion mechanisms (Moriya et al. 2014), and environments of explosion sites (Habergham et al. 2014; Taddia et al. 2015).

The fractional population of Type IIn $\mathrm{SNe}$ is relatively small among the volume limited survey of CCSNe $(\sim 10$ per cent, Li et al. 2011). Nonetheless, considering their peculiarities, the light curve and spectral evolution of several events have been studied in detail. The polarimetric (broad-band polarimetry and spectropolarimetry) investigations of Type IIn SNe in the past have suggested relatively 
high degree of polarization in comparison to other CCSNe. In addition, they do not exhibit significant variation in the polarization angle (cf. Wang \& Wheeler 2008; Branch \& Wheeler 2017, and references therein). This is attributed to the intrinsic aspherical SN explosion and/or asphericity in the CSM. The degree of polarization varies during different phases of the $\mathrm{SN}$ evolution [for example, SN 1994Y (Wang et al. 1996), SN 1997eg (Hoffman et al. 2008), SN 1998S (Leonard et al. 2000; Wang et al. 2001), SN 2010j1 (Patat et al. 2011), and SN 2012ab (Bilinski et al. 2018)]. While spectropolarimetry serves as a direct geometrical and dynamical probe of chemical constituents and geometry of the SN ejecta (see e.g. Shapiro \& Sutherland 1982), the broad-band polarization parameters, on the other hand, provide a coarse but important picture of $\mathrm{SN}$, especially when it is fainter.

Emergence of $\sim 1$ percent intrinsic polarization may imply around 20 per cent asymmetry (Hoflich 1991; Leonard \& Filippenko 2005). In the case of Type IIn SNe, the theoretical interpretation of the observed polarization is not straightforward because of the difficulties in disentangling the polarization contributors. It is suggested that geometry of the SN ejecta along with the geometry of the CSM are to be accounted (Chugai 2001). There is an increasing need for additional polarimetric data of SNe in order to enhance our current understanding about the structure of SN explosion/ejecta. Multi-epoch broad-band polarization and spectroscopic observations of SN 2017hcc are presented here with an investigation of its observational properties.

SN 2017hcc (ATLAS 17lsn) was discovered ${ }^{2}$ ( 17.4 mag, $o$ band) in an anonymous galaxy on 2017 October 2.4 (JD 2458028.9) by Asteroid Terrestrial-impact Last Alert System (ATLAS, Tonry 2011). It was located 4.3 arcsec east and 0.5 arcsec south of the centre of the host galaxy, with coordinates $\alpha=00^{\mathrm{h}} 03^{\mathrm{m}} 50 \mathrm{~s} .6, \delta=$ $-11^{\circ} 28^{\prime} 28^{\prime \prime} .8$. The transient was not detected on 2017 September 30.4 (JD 2458026.9) at a limiting magnitude of 19.04. This indicates that $\mathrm{SN}$ was discovered within a few days after the explosion. In the present study, we adopted a mean of the earliest detection and the last non-detection epochs as the date of explosion for SN $2017 \mathrm{hcc}$ i.e. 2017 October 01.4 (JD 2458027.9). It was classified ${ }^{3}$ as a young Type IIn SN based on a low-resolution optical spectrum obtained on 2017 October 07 with FLOYDS spectrograph at Siding Spring Observatory. In subsequent observations, non-detection of X-ray and radio was reported by Chandra, Fransson \& Chevalier (2017) and Nayana \& Chandra (2017), respectively.

Spectropolarimetric observations of SN 2017hcc obtained on 2017 October 30 by Mauerhan et al. (2017b) indicated an integrated $V$-band continuum polarization of 4.84 per cent with a polarization angle of $\sim 97^{\circ}$. They also noticed a weak wavelength dependence in the polarization measurement and a strong depolarization in the cores of the narrow emission features of $\mathrm{H} \alpha$ and $\mathrm{H} \beta$. Prieto et al. (2017) reported early phase results of SN 2017hcc and found it to be one of the brightest Type IIn events known $\left(M_{\mathrm{V}} \sim-20.7 \mathrm{mag}\right.$ and peak $L_{\mathrm{bol}} \sim 1.3 \times 10^{44} \mathrm{erg} \mathrm{s}^{-1}$, for a distance of about $73 \mathrm{Mpc}$ ).

\section{OBSERVATIONS AND DATA REDUCTION}

The $V$-band $\left(\lambda_{V_{\text {eff }}}=0.53 \mu \mathrm{m}\right)$ polarimetric data of SN $2017 \mathrm{hcc}$ was obtained on 2017 December 25. These observations were acquired using the ARIES Imaging Polarimeter (AIMPOL, Rautela, Joshi \& Pandey 2004) mounted at the Cassegrain focus of the

\footnotetext{
${ }^{2}$ https://wis-tns.weizmann.ac.il/object/2017hcc/discovery-cert
}

${ }^{3}$ https://wis-tns.weizmann.ac.il/object/2017hcc/classification-cert

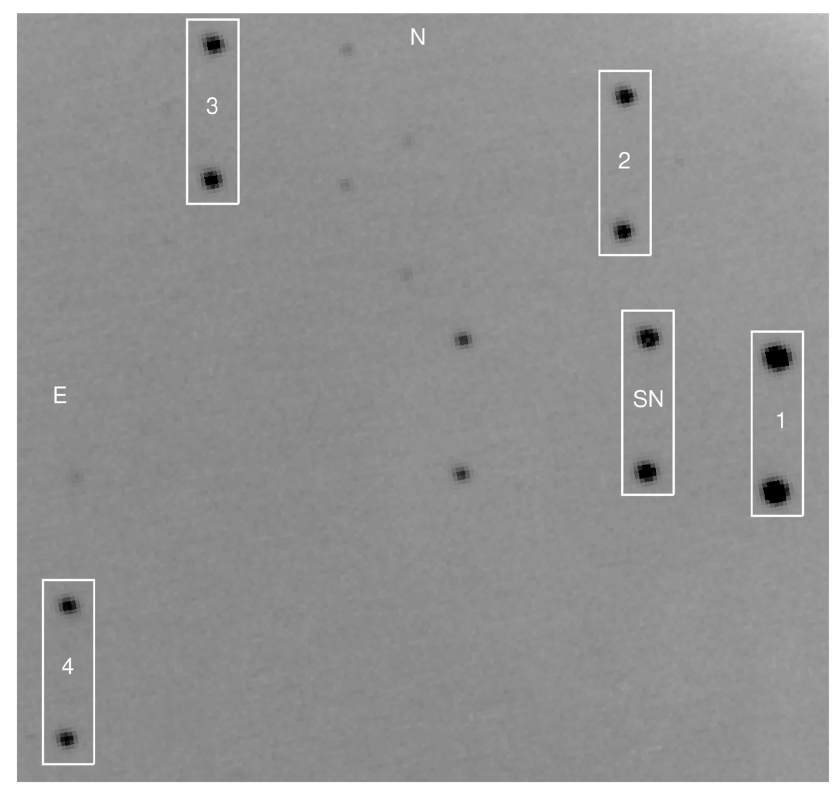

Figure 1. SN 2017hcc and field stars (1-4). A zoomed AIMPOL $V$-band image (about 4.5 arcmin $\times 4.5$ arcmin) obtained with the ST on 2017 December 25 is shown. Each object has two images (i.e. ordinary and extraordinary) and are marked inside rectangular boxes. North and east directions are indicated.

1.04-m Sampurnanand telescope (ST) at Manora Peak, Nainital. This observing facility is operated by the Aryabhatta Research Institute of Observational Sciences (ARIES), India. A half-wave plate (HWP) modulator, a Wollaston prism beam-splitter and a liquid nitrogen cooled Tektronix $1 \mathrm{k} \times 1 \mathrm{k}$ pixel CCD camera are integrated in the AIMPOL instrument. The plate scale, readout noise and gain of the CCD are 1.73 arcsec pixel $^{-1}, 7.0 e^{-}$, and 11.98 $e^{-} \mathrm{ADU}^{-1}$, respectively. The central 370 pixel $\times 370$ pixel region of the CCD, used for observation, covers $\sim 8$ arcmin in diameter in the sky. Fig. 1 displays a section (approximately $4.5 \operatorname{arcmin} \times$ $4.5 \mathrm{arcmin}$ ) of the full image captured with the AIMPOL using ST. AIMPOL has been effectively used for polarimetric observations of SNe, e.g., SN 2012aw (Kumar et al. 2014), SN 2013ej (Kumar et al. 2016), SN 2013hj and SN 2014G (Bose et al. 2016), SN 2014J (Srivastav et al. 2016) and SN 2016B (Dastidar et al. 2019), etc.

Five frames were secured at each position angle of the HWP (i.e. $0.0^{\circ}, 22.5^{\circ}, 45.0^{\circ}$, and $67.5^{\circ}$ ). The individual exposure time for each frame was $200 \mathrm{~s}$. To obtain a good signal-to-noise ratio $(\mathrm{S} / \mathrm{N})$, all pre-processed images at a given position angle were aligned and subsequently combined. The ordinary and extraordinary fluxes were extracted by performing standard aperture photometry using $\mathrm{IRAF}^{4}$ software. Multiple apertures (2-8 pixels) were chosen to estimate Stokes parameters at each aperture and finally $P$ (degree of polarization) and $\theta$ (polarization angle) were computed. Detailed description of the various procedure and the requisite equations can be found in Ramaprakash et al. (1998), Rautela et al. (2004), and Kumar et al. $(2014,2016)$. The considered $P$ and $\theta$ values are for the aperture that best fit with minimum chi-square.

\footnotetext{
${ }^{4}$ IRAF is the Image Reduction and Analysis Facility distributed by the National Optical Astronomy Observatories, which are operated by the Association of Universities for Research in Astronomy, Inc., under cooperative agreement with the National Science Foundation.
} 
Table 1. Results of observed polarized and unpolarized standard stars taken from Schmidt, Elston \& Lupie (1992).

\begin{tabular}{|c|c|c|c|}
\hline $\begin{array}{l}\text { Standard star } \\
\text { (ID) }\end{array}$ & Band & $\begin{array}{c}P \pm \sigma_{P} \\
\text { (per cent) }\end{array}$ & $\begin{array}{c}\theta \pm \sigma_{\theta} \\
\left(^{\circ}\right)\end{array}$ \\
\hline $\mathrm{BD}+59^{\circ} 389(\mathrm{Pol})^{a}$ & $R$ & & \\
\hline Standard values & & $6.43 \pm 0.02$ & $98.14 \pm 0.10$ \\
\hline Observed values & & $6.49 \pm 0.02$ & $98.40 \pm 0.06$ \\
\hline $\mathrm{BD}+64^{\circ} 106(\mathrm{Pol})^{a}$ & $R$ & & \\
\hline Standard values & & $5.15 \pm 0.10$ & $96.74 \pm 0.54$ \\
\hline Observed values & & $5.35 \pm 0.02$ & $97.70 \pm 0.17$ \\
\hline HD $25443(\mathrm{Pol})^{b}$ & V & & \\
\hline Standard values & & $5.13 \pm 0.06$ & $134.23 \pm 0.34$ \\
\hline Observed values & & $5.50 \pm 0.10$ & $144.60 \pm 1.00$ \\
\hline HD $236633(\mathrm{Pol})^{b}$ & V & & \\
\hline Standard values & & $5.49 \pm 0.02$ & $93.76 \pm 0.08$ \\
\hline Observed values & & $5.40 \pm 0.10$ & $103.5 \pm 2.00$ \\
\hline HD $12021(\text { Unpol })^{b}$ & V & & \\
\hline Standard values & & $0.08 \pm 0.02$ & $160.1-$ \\
\hline Observed values & & $0.12 \pm 0.10$ & $163.8 \pm 3.50$ \\
\hline
\end{tabular}

Notes. ${ }^{a} \mathrm{HONIR}$.

${ }^{b}$ AIMPOL.

The offset polarization angle was estimated by observing two polarized standard stars on the same night. For this purpose, HD 25443 and HD 236633 stars were selected from Schmidt, Elston \& Lupie (1992). The results listed in Table 1 are in good agreement with the standard values. Consequently, an average of offset values (i.e. $10.1^{\circ}$ ) was applied to the $\mathrm{SN}$. The instrumental polarization of AIMPOL is generally found to be small, at $\sim 0.1$ per cent (see e.g. Medhi et al. 2007; Pandey et al. 2009; Das et al. 2013; Eswaraiah et al. 2012, 2013; Patel et al. 2013; Soam et al. 2015; Neha et al. 2016; Rakshit et al. 2017; Wang et al. 2017; Lee et al. 2018, and references therein). This was verified by the observations of the unpolarized standard star HD 12021 (Schmidt, Elston \& Lupie 1992). The observed $P$ and $\theta$ values of SN 2017hcc after correcting for the offset polarization angle and instrumental polarization are provided in Table 4.

Imaging polarimetric observations of SN 2017hcc were also made using the Hiroshima Optical and Near-InfraRed camera (HONIR; Akitaya et al. 2014) mounted on the 1.5-m Kanata telescope at Higashi-Hiroshima Observatory, in the $R$ band $\left(\lambda_{R_{\text {eff }}}=0.65\right.$ $\mu \mathrm{m})$ on 2017 November 9.5 and December 22.5. The observations consisted of a sequence of exposures at four position angles of the achromatic HWP (i.e. $0.0^{\circ}, 45.0^{\circ}, 22.5^{\circ}$, and $67.5^{\circ}$ ). The position angle of the polarization was calibrated using the observations of the polarized standard stars $\left(\mathrm{BD}+59^{\circ} 389, \mathrm{BD}+64^{\circ} 106\right.$; Schmidt et al. 1992) as listed in Table 1. Since the instrumental polarization was estimated to be negligible ( $\sim 0.02$ percent $)$, it has not been corrected for.

The Ultra Violet Optical Telescope (UVOT) onboard the Swift satellite monitored SN 2017hcc from JD 2458054.9 to 2458099.8 in $u v w 2, u v m 2, u v w 1, u, b$, and $v$ bands (see Poole et al. 2008, for filter specifications). These data sets were retrieved from the Swift data archive. To estimate the SN magnitude in each band, aperture photometry was performed using various modules of the HEASOFT (High Energy Astrophysics Software). A detailed description can be found in Kumar et al. (2018) and Sahu et al. (2018). The UVOT magnitudes (in Vega system) are listed in Table 2.

We obtained a low-resolution spectrum of SN 2017hcc with the Himalayan Faint Object Spectrograph Camera (HFOSC), mounted on the f/9 Cassegrain focus of the 2-m Himalayan Chandra Telescope (HCT) of Indian Astronomical Observatory (IAO), Hanle, India. Observations were performed on 2017 November 23 ( $\sim 27$ d post-maximum) using two grisms Gr\#7 (3500-7800 $\AA$ ) and Gr\#8 (5200-9250 A), having a resolution of $\sim 7 \AA$. A description on the data reduction procedure can be found in our previous studies (e.g. Kumar et al. 2018; Sahu et al. 2018; Singh et al. 2018, 2019). The spectrum was brought to an absolute flux scale using the blackbody fit to the extinction $(E(B-V)=0.029$ mag) corrected Swift-UVOT fluxes.

\section{RESULTS AND DISCUSSION}

In this section, we constrain the interstellar polarization (ISP) and derive the intrinsic polarization characteristics of SN 2017hcc. The evolution of light curves, spectral properties, and physical parameters are also discussed.

\subsection{Interstellar polarization}

The ISP originates [both due to the Milky Way (MW) and/or the host galaxy] owing to directional extinction of the dust grains situated along the line of sight (LOS) of an observer. Accordingly, the intrinsic signal of a source gets altered when it passes through the interstellar medium. The situation becomes more complicated in case of extragalactic $\mathrm{SNe}$ as the host galaxy dust and local

Table 2. The Swift-UVOT photometric magnitudes of SN 2017hcc (in Vega system).

\begin{tabular}{|c|c|c|c|c|c|c|c|c|}
\hline $\begin{array}{l}\text { Date } \\
\text { (yyyy-mm-dd) }\end{array}$ & $\begin{array}{c}\text { JD } \\
2458000+\end{array}$ & Phase $^{a}$ & $\begin{array}{c}u \\
(\mathrm{mag})\end{array}$ & $\begin{array}{c}b \\
(\mathrm{mag})\end{array}$ & $\begin{array}{c}v \\
(\mathrm{mag})\end{array}$ & $\begin{array}{l}\text { uvm2 } \\
\text { (mag) }\end{array}$ & $\begin{array}{l}u v w 1 \\
(\mathrm{mag})\end{array}$ & $\begin{array}{l}u v w 2 \\
(\mathrm{mag})\end{array}$ \\
\hline 2017-10-28 & 54.9 & +0.4 & $12.72 \pm 0.03$ & $13.98 \pm 0.03$ & $13.92 \pm 0.03$ & $12.27 \pm 0.03$ & $12.33 \pm 0.02$ & $12.43 \pm 0.02$ \\
\hline 2017-11-03 & 61.3 & +6.8 & $12.62 \pm 0.03$ & $13.81 \pm 0.03$ & $13.78 \pm 0.03$ & $12.33 \pm 0.02$ & $12.34 \pm 0.02$ & $12.52 \pm 0.02$ \\
\hline 2017-11-05 & 63.3 & +8.8 & $12.60 \pm 0.03$ & $13.80 \pm 0.03$ & $13.72 \pm 0.04$ & $12.37 \pm 0.03$ & $12.37 \pm 0.03$ & $12.59 \pm 0.02$ \\
\hline 2017-11-10 & 68.0 & +13.5 & $12.61 \pm 0.03$ & $13.81 \pm 0.03$ & $13.72 \pm 0.04$ & $12.48 \pm 0.03$ & $12.44 \pm 0.03$ & $12.73 \pm 0.02$ \\
\hline $2017-11-22$ & 80.1 & +25.6 & $12.77 \pm 0.04$ & $13.77 \pm 0.03$ & $13.58 \pm 0.04$ & $12.96 \pm 0.03$ & $12.81 \pm 0.03$ & $13.23 \pm 0.03$ \\
\hline $2017-11-30$ & 87.8 & +33.3 & $12.92 \pm 0.04$ & $13.86 \pm 0.04$ & $13.61 \pm 0.05$ & $13.42 \pm 0.03$ & $13.19 \pm 0.03$ & $13.69 \pm 0.03$ \\
\hline 2017-12-04 & 92.1 & +37.6 & $13.13 \pm 0.03$ & $13.94 \pm 0.03$ & $13.70 \pm 0.03$ & $13.71 \pm 0.03$ & $13.42 \pm 0.03$ & $13.95 \pm 0.03$ \\
\hline 2017-12-08 & 95.7 & +41.2 & $13.22 \pm 0.04$ & $13.98 \pm 0.03$ & $13.68 \pm 0.05$ & - & $13.67 \pm 0.04$ & $14.25 \pm 0.04$ \\
\hline 2017-12-12 & 99.8 & +45.3 & $13.41 \pm 0.03$ & $14.07 \pm 0.03$ & $13.73 \pm 0.04$ & $14.24 \pm 0.03$ & $13.84 \pm 0.03$ & $14.51 \pm 0.03$ \\
\hline
\end{tabular}

Note. ${ }^{a}$ With reference to the maximum light JD 2458054.5. 
Table 3. Details of 17 isolated field stars within $5^{\circ}$ radius around the SN which are used to constrain the ISP (Section 3.1 ). Listed distances are taken from Gaia DR2 catalogue. The mentioned polarization values for HD and $\mathrm{S}_{1-4}$ star IDs are from Heiles catalogue and estimated from AIMPOL observations, respectively.

\begin{tabular}{|c|c|c|c|c|c|c|c|}
\hline $\begin{array}{l}\text { Star } \\
\text { ID }\end{array}$ & $\begin{array}{c}\text { RA (J2000) } \\
\text { (h:m:s) }\end{array}$ & $\begin{array}{l}\text { Dec. }(\mathrm{J} 2000) \\
\quad(\mathrm{d}: \mathrm{m}: \mathrm{s})\end{array}$ & $\begin{array}{l}\text { Distance } \\
\quad(\mathrm{pc})\end{array}$ & $\begin{array}{l}P_{\mathrm{V}} \pm \sigma_{P_{\mathrm{V}}} \\
\text { (per cent) }\end{array}$ & $\begin{array}{c}\theta_{\mathrm{V}} \pm \sigma_{\theta_{\mathrm{V}}} \\
\left(^{\circ}\right)\end{array}$ & $\begin{array}{c}Q \\
\text { (per cent) }\end{array}$ & $\begin{array}{c}U \\
\text { (per cent) }\end{array}$ \\
\hline HD 693 & $00: 11: 16.1$ & $-15: 27: 51.1$ & $17.99 \pm 0.11$ & $0.01 \pm 0.01$ & $135 \pm 29$ & $-0.0000 \pm 0.0082$ & $-0.0080 \pm 0.0090$ \\
\hline HD 1388 & $00: 17: 58.6$ & $-13: 27: 20.2$ & $26.94 \pm 0.04$ & $0.02 \pm 0.02$ & $136 \pm 25$ & $0.0003 \pm 0.0168$ & $-0.0190 \pm 0.0180$ \\
\hline HD 967 & $00: 14: 04.2$ & $-11: 18: 40.0$ & $42.84 \pm 0.10$ & $0.11 \pm 0.05$ & $108 \pm 13$ & $-0.0868 \pm 0.0507$ & $-0.0626 \pm 0.0504$ \\
\hline HD 224383 & $23: 57: 33.3$ & $-09: 38: 49.6$ & $51.37 \pm 0.17$ & $0.02 \pm 0.03$ & $115 \pm 41$ & $-0.0102 \pm 0.0252$ & $-0.0123 \pm 0.0261$ \\
\hline HD 1195 & $00: 16: 18.6$ & $-14: 28: 18.8$ & $79.11 \pm 0.44$ & $0.02 \pm 0.01$ & $134 \pm 12$ & $-0.0007 \pm 0.0089$ & $-0.0210 \pm 0.0090$ \\
\hline HD 1064 & $00: 14: 54.5$ & $-09: 34: 10.2$ & $110.49 \pm 1.36$ & $0.07 \pm 0.01$ & $141 \pm 5$ & $0.0136 \pm 0.0111$ & $-0.0676 \pm 0.0110$ \\
\hline HD 223774 & $23: 52: 30.1$ & $-14: 15: 04.3$ & $112.70 \pm 1.97$ & $0.11 \pm 0.06$ & $150 \pm 14$ & $0.0553 \pm 0.0570$ & $-0.0974 \pm 0.0577$ \\
\hline HD 604 & $00: 10: 26.1$ & $-14: 27: 05.0$ & $132.17 \pm 0.89$ & $0.09 \pm 0.01$ & $135 \pm 4$ & $0.0010 \pm 0.0140$ & $-0.0910 \pm 0.0140$ \\
\hline HD 1231 & 001634.1 & $-13: 24: 12.2$ & $150.19 \pm 1.39$ & $0.11 \pm 0.01$ & $17 \pm 3$ & $0.0924 \pm 0.0120$ & $0.0633 \pm 0.0121$ \\
\hline HD 224481 & $23: 58: 21.2$ & $-15: 50: 50.6$ & $164.00 \pm 1.91$ & $0.10 \pm 0.04$ & $121 \pm 10$ & $-0.0469 \pm 0.0347$ & $-0.0883 \pm 0.0349$ \\
\hline HD 223559 & $23: 50: 33.3$ & $-14: 24: 05.0$ & $181.42 \pm 6.67$ & $0.17 \pm 0.03$ & $147 \pm 6$ & $0.0667 \pm 0.0340$ & $-0.1542 \pm 0.0340$ \\
\hline HD 1114 & $00: 15: 24.4$ & $-13: 03: 15.1$ & $210.34 \pm 2.54$ & $0.16 \pm 0.02$ & $136 \pm 3$ & $0.0079 \pm 0.0170$ & $-0.1618 \pm 0.0170$ \\
\hline HD 1268 & $00: 16: 52.9$ & $-14: 43: 03.7$ & $259.07 \pm 5.85$ & $0.16 \pm 0.02$ & $145 \pm 3$ & $0.0529 \pm 0.0169$ & $-0.1520 \pm 0.0170$ \\
\hline $\mathrm{S}_{1}{ }^{a}$ & $00: 03: 47.5$ & $-11: 28: 35.1$ & $3640.3 \pm 551.3$ & $0.52 \pm 0.02$ & $159 \pm 1$ & $0.3822 \pm 0.0184$ & $-0.3482 \pm 0.0181$ \\
\hline $\mathrm{S}_{2}{ }^{a}$ & 00:03:51.2 & $-11: 27: 04.4$ & $1588.1 \pm 86.2$ & $0.29 \pm 0.21$ & $61 \pm 15$ & $-0.1519 \pm 0.1728$ & $0.2517 \pm 0.1980$ \\
\hline $\mathrm{S}_{3}{ }^{a}$ & 00:04:00.9 & $-11: 26: 47.7$ & $549.1 \pm 9.9$ & $0.24 \pm 0.22$ & $5 \pm 18$ & $0.2364 \pm 0.2144$ & $0.0415 \pm 0.1544$ \\
\hline $\mathrm{S}_{4}{ }^{a}$ & $00: 04: 04.3$ & $-11: 30: 02.6$ & $1843.0 \pm 131.1$ & $0.65 \pm 0.39$ & $165 \pm 15$ & $0.5647 \pm 0.3773$ & $-0.3260 \pm 0.3483$ \\
\hline
\end{tabular}

Note. ${ }^{a}$ Field stars around SN 2017hcc within the FOV of AIMPOL.

environment may further contaminate the propagating signal. Consequently, the intervening ISP contribution should be accounted for from the observed SN measurements in order to derive the intrinsic polarization characteristics of $\mathrm{SN}$. While temporal variation may be observed in the SN polarization properties, the ISP should be constant towards a particular direction. Therefore, if ISP can be constrained appropriately (or at-least setting an upper limit), it will be easier to estimate intrinsic SN parameters by subtracting it from the observed values. Fortunately, the Galactic ISP has been properly documented in literature (e.g. Serkowski, Mathewson \& Ford 1975; Whittet \& van Breda 1978).

In order to examine the ISP towards the $\mathrm{SN}$ arising due to the MW, the nearby field stars (well above the Galactic plane) can serve as good probe (Tran 1995). For this purpose, we selected 13 isolated field stars within a radius of $5^{\circ}$ around the location of SN 2017hcc which do not show emission, variability, and double characteristics in SIMBAD database. The polarization and distance information of these sources were collected from the polarization data catalog of Heiles (2000) and the newly available Gaia data (DR2) (Gaia Collaboration et al. 2018), respectively. Additionally, four stars (indicated with IDs 1-4 in Fig. 1) lying within the 8 arcmin AIMPOL field of SN 2017hcc were also considered. Different parameters of all 17 stars are listed in Table 3 and their $P_{\mathrm{V}}$ and $\theta_{\mathrm{V}}$ measurements are plotted against distance in Fig. 2 (left-hand panel). An increasing trend can be seen in the $P_{\mathrm{V}}$ values as a function of distance up to $\sim 260 \mathrm{pc}$. The $\theta_{\mathrm{V}}$ values are generally distributed between $100^{\circ}$ and $150^{\circ}$ (except for a few sources).

The $P_{\mathrm{V}}$ and $\theta_{\mathrm{V}}$ of the field stars were converted into Stokes parameters $(Q$ and $U)$ using the relations $Q_{\mathrm{V}}=P_{\mathrm{V}} \cos \left(2 \theta_{\mathrm{V}}\right)$ and $U_{\mathrm{V}}=P_{\mathrm{V}} \sin \left(2 \theta_{\mathrm{V}}\right)$. The distribution of $Q_{\mathrm{V}}$ and $U_{\mathrm{V}}$ with respect to distance is shown in Fig. 2 (right-hand panel). Further, weighted linear fits on the distance versus Stokes parameters were performed as shown with dotted lines in the same figure. It is to be noted that the Heiles catalogue stars have reliable information on their spectral types. However, this is not the case for field stars situated in the field of view (FOV) of AIMPOL (except for the RR Lyrae variable, S1, Gavrilchenko et al. 2014). Stars S2, S3, and S4 are faint and have large polarization error bars due to low S/N. HD 1231 is deviant amongst the Heiles catalogue stars. Consequently, these stars (S1S4 and HD 1231) were excluded from the fitting.

The fitted slopes and intercepts at the distance $260 \mathrm{pc}$ of HD 1268 were used to estimate the Stokes parameters $Q_{\mathrm{ISP}_{\mathrm{MW}}}$ and $U_{\mathrm{ISP}_{\mathrm{MW}}}$ values corresponding to the ISP contribution in the MW. From these parameters, the derived $P_{\mathrm{ISP}_{\mathrm{MW}}}$ and $\theta_{\mathrm{ISP}}$ were found to be $0.17 \pm 0.02$ per cent and $140.3^{\circ} \pm 3.0^{\circ}$, respectively.

The MW reddening in the direction of SN 2017hcc was estimated as $E(B-V)=0.029 \pm 0.001$ mag using the all-sky dust extinction map (Schlafly \& Finkbeiner 2011). Polarimetric studies of Galactic stars along different directions have provided a correlation between ISP and reddening (e.g. Serkowski et al. 1975). According to this, the mean and maximum polarization efficiency are correlated with interstellar extinction as $P_{\text {mean }}=5 \times E(B-V)$ and $P_{\max }=9 \times E(B$ $-V$ ), respectively. Assuming that MW dust grains follow mean polarization efficiency along the $\mathrm{SN}$ direction, the first relation yields $P_{\text {mean }}$ of 0.15 per cent. This value is similar to the ISP value ( $P_{\mathrm{ISP}}=0.17$ per cent $)$ computed using the polarization measured for the field stars. Using the maximum polarization efficiency relation, an upper limit of i.e. $P_{\max }=0.26$ per cent is obtained.

The 3D dust extinction map $^{5}$ (Green et al. 2018) was used to further explore the MW dust extinction along the LOS towards SN 2017hcc. The map is based on high-quality stellar photometry data from Pan-STARRS and 2MASS surveys, and provides information of $E(B-V)$ as a function of distance. The extinction map indicates the best-fitting reddening value remains constant around 0.033 mag beyond a distance of $650 \mathrm{pc}$. This implies the predicted degree of polarization, as per the mean polarization efficiency relation, would be 0.17 per cent. This again confirms our ISP estimate using the weighted linear fits on distance versus Stokes parameters.

The colour excess of the host galaxy can be inferred using the equivalent width (EW) of Na I D absorption lines (Turatto, Benetti \& Cappellaro 2003; Poznanski, Prochaska \& Bloom 2012; Stritzinger et al. 2018). This signature is very weak at the redshift of the

\footnotetext{
${ }^{5}$ http://argonaut.skymaps.info/
} 

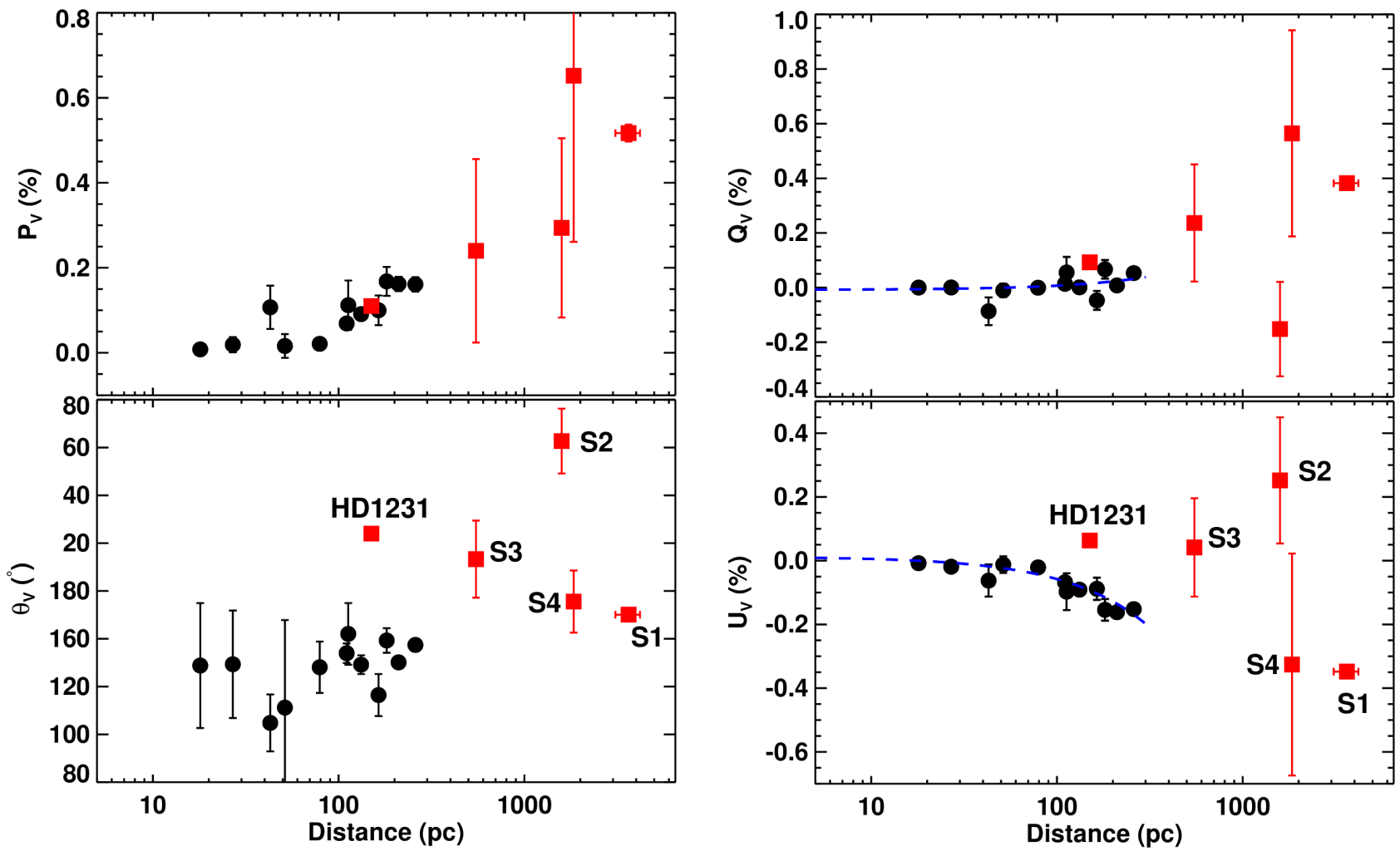

Figure 2. Distribution of polarization parameters of 17 field stars around SN 2017hcc. Left-hand panel: $P_{\mathrm{V}}$ and $\theta_{\mathrm{V}}$ are plotted against distance in top and bottom panels, respectively. The polarization values are from Heiles (2000, dot symbols and HD 1231) and AIMPOL ( $\left.S_{1-4}\right)$. Parallax measurements are from Gaia Collaboration et al. (2018). Right-hand panel: $Q_{\mathrm{V}}$ and $U_{\mathrm{V}}$ plots for same field stars. In each panel, dashed lines are the weighted linear fits on the Stokes parameters versus distances. Notably, HD 1231 and $\mathrm{S}_{1-4}$ (indicated with filled squares) were not used in the fits (see Section 3.1).

host galaxy in the low-resolution spectrum presented here (see inset in Fig. 4). We estimate an upper limit of $0.06 \AA$ on Na I D EW, indicating negligible host extinction. Mauerhan et al. (2017b) have made a similar observation based on spectropolarimetric data obtained close to maximum light.

The direct and indirect methods discussed above, indicate that the MW aligned dust grains exhibit mean polarization efficiency. Therefore, in the present analysis we have considered only MW dust as the major source of ISP along the LOS of SN 2017hcc. An average ISP as a function of wavelength was estimated using the formulation of Serkowski et al. (1975) and found to be similar for both $V$ and $R$ bands $\left(P_{\mathrm{ISP}}=0.17\right.$ percent and $\left.\theta_{\text {ISP }}=140.3^{\circ}\right)$. The corresponding $Q_{\text {ISP }}$ and $U_{\text {ISP }}$ values are $0.032 \pm 0.018$ and $-0.170 \pm 0.019$ per cent which are subtracted from the observed SN polarization measurements (see Section 3.4). A similar methodology has been used to derive the ISP contribution in B[e] stars and dark globules (Lee et al. 2018; Eswaraiah et al. 2019).

\subsection{Light curves and spectral features}

The Swift-UVOT light curves of SN 2017hcc are displayed in Fig. 3. We adopt JD 2458054.5 as the epoch of maximum from Prieto et al. (2017) as inferred from their bolometric light curve. The $b$ - and $v$-band light curves are almost flat compared to the bluer bands. The decay rates (between 0 and $45 \mathrm{~d}$ ) in $u, u v w 1, u v w 2$, and $u v m 2$ bands are $\sim 0.02,0.04,0.05$, and $0.05 \mathrm{mag} \mathrm{d}^{-1}$, respectively.

In Fig. 4, the redshift corrected $(z=0.0168$, Prieto et al. 2017) spectrum of SN 2017hcc, obtained on 2017 November $23(\sim+27$

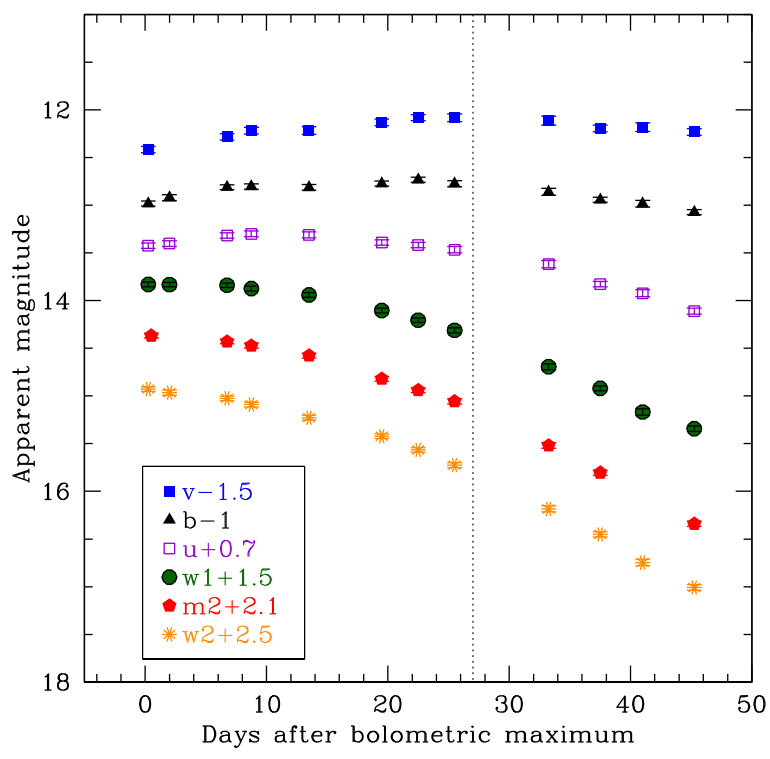

Figure 3. Evolution of Swift-UVOT light curves. Vertical shift with indicated amount has been applied for clarity. A dotted line indicates epoch of spectrum taken with the HCT (see Sections 2 and 3.2).

d) is displayed. Spectra of SN 2010jl (Zhang et al. 2012) and SN 2012ab (Gangopadhyay et al. 2019), at a similar epoch are also shown in the figure for comparison. It is evident from the figure that the spectrum of SN 2017hcc is considerably bluer in 


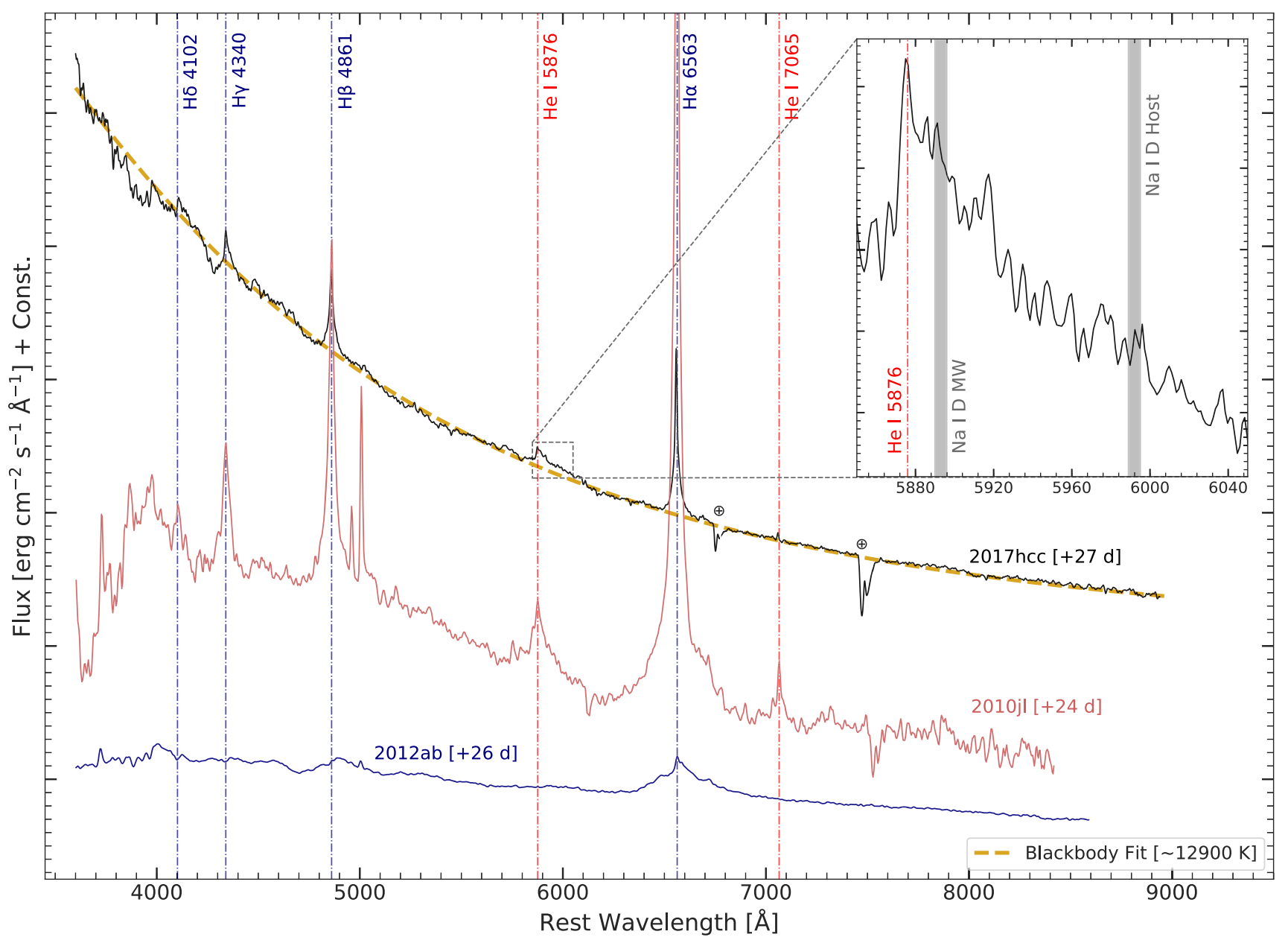

Figure 4. Comparison of SN 2017hcc, SN 2012ab (Gangopadhyay et al. 2019), and SN 2010jl (Zhang et al. 2012) spectra at similar epochs. Prominent Balmer and $\mathrm{He}$ I lines are also marked. The encircled plus symbols indicate telluric lines. The spectra have been corrected for the respective host galaxy redshift. Given phases are relative to maximum light. The blackbody model fit to SN 2017hcc is shown with dotted line (see Section 3.2). A zoomed spectrum region (5850-6050 ̊̊) of SN 2017hcc is shown in the inset.

comparison to the other two events. Prieto et al. (2017) obtained a spectrum of SN 2017 hcc on 2017 October $20(\sim+8$ d) which also reported a significantly bluer continuum. The primary spectral features are also marked in Fig. 4. The emission lines of hydrogen i.e. $\mathrm{H} \alpha(6563 \AA), \mathrm{H} \beta$ (4861 $\AA$ ), $\mathrm{H} \gamma(4341 \AA)$, and $\mathrm{H} \delta(4102 \AA)$ are prominent in all the three events but the $\mathrm{H} \alpha$ and $\mathrm{H} \beta$ lines are broader in SN 2012ab and SN 2010jl compared to SN 2017hcc. The blackbody fit to the continuum indicates a temperature of $\sim 12900 \pm 680 \mathrm{~K}$ (shown with a dotted line in Fig. 4). The flux ratio of $\mathrm{H} \alpha$ to $\mathrm{H} \beta$ lines is estimated as $\sim 4$ for $\mathrm{SN} 2017 \mathrm{hcc}$, whereas the same ratio is found to be $\sim 5$ and $\sim 8$ for SN $2010 \mathrm{jl}$ and $\mathrm{SN} 2012 \mathrm{ab}$, respectively. The $\mathrm{H} \alpha$ line profile is decomposed into narrow and broad components by fitting a two-component Gaussian as shown in Fig. 5. The best fit yields a full width at half-maximum of 306 and $1862 \mathrm{~km} \mathrm{~s}^{-1}$ for the narrow and broad components, respectively.

The He I 5876, $7065 \AA$ features are stronger in SN 2017hcc and SN 2010jl than in SN 2012ab. An unblended signature of a Na I D feature was found in SN 2012ab (Bilinski et al. 2018) which is negligible in SN 2017hcc and SN 2010jl (Zhang et al. 2012) indicating negligible reddening (see Section 3.1) along the LOS caused by the host galaxies.

\subsection{Basic physical parameters}

The temperature and radius evolution of SN 2017hcc was computed by fitting a blackbody function to the spectral energy distribution (SED) constructed using the Swift magnitudes (cf. Table 2). Weighted least-squares optimization was employed for the fit with the weights being the inverse of the $1 \sigma$ error on the Swift magnitudes. The evolution of these parameters are shown in Fig. 6. The temperature at the earliest epoch $(\sim 0 \mathrm{~d})$, estimated at $\sim 16000 \mathrm{~K}$ is similar to the temperature obtained in Prieto et al. (2017). The temperature subsequently decreased to $\sim 8700 \mathrm{~K}$ at the last epoch $(\sim+45 \mathrm{~d})$. In comparison, SN 2012ab had a temperature of $\sim 12000 \mathrm{~K}$ at $-22 \mathrm{~d}$, which decreased to $\sim 10500 \mathrm{~K}$ close to the maximum. The peculiar Type IIn SN 2006gy displayed a temperature of 12000 K (Smith et al. 2007), whereas SN 2006tf reached a temperature of $\sim 7800 \mathrm{~K}$ near the peak (Smith et al. 2008).

The temperature evolution of SN 2017hcc clearly indicates that it is hotter than other Type IIn events at similar epochs. The blackbody radius of Type IIn SNe is found to steadily increase with time, reach a peak and subsequently decline, as observed in several events like SN 1998S (Fassia et al. 2001), SN 2005gj (Prieto et al. 2007), and SN 2006gy (Smith et al. 2010). The peak represents a transition to the optically thin CSM shell (Smith et al. 2010). In SN 2017hcc, 


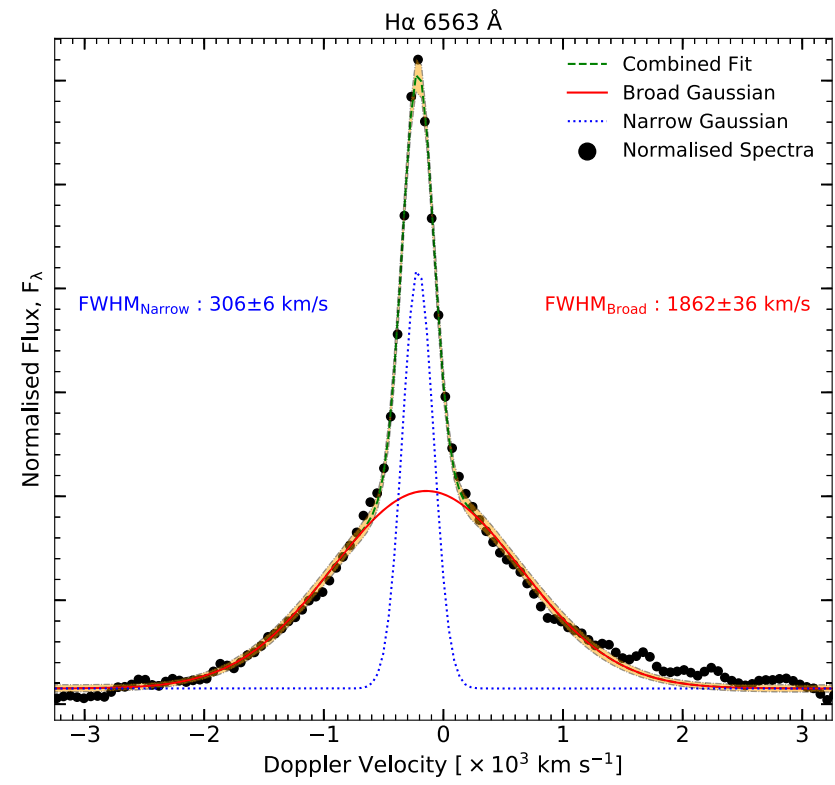

Figure 5. Gaussian fit to the observed spectrum of SN 2017hcc. The $3 \sigma$ confidence interval of the fit has been indicated with an orange shaded region.

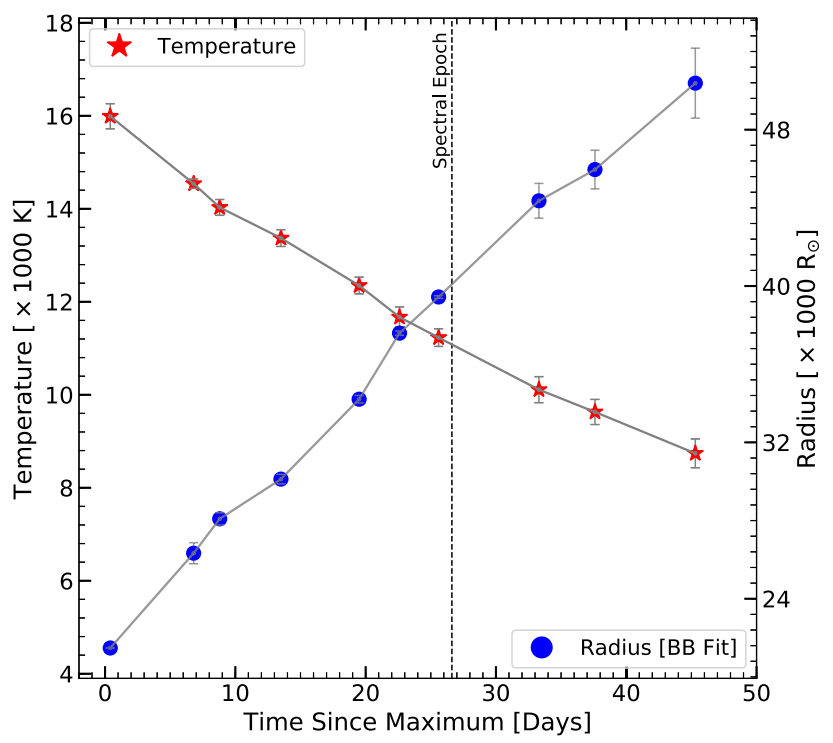

Figure 6. Temperature and radius evolution of SN 2017hcc.

the blackbody radius was found to be increasing until the last epoch of Swift observation (cf. Fig. 6) which is likely due to the optically thick $\mathrm{SN}$ atmosphere until day +45 .

The overall light-curve evolution and spectral features suggests the presence of CSM interaction in SN 2017hcc. Assuming that the luminosity is mainly powered by the ejecta-CSM interaction (Chevalier \& Fransson 1994), the mass-loss rate $(\dot{M})$ can be estimated using the relation of Chugai \& Danziger (1994):

$\dot{M}=\frac{2 L}{\epsilon} \frac{v_{\mathrm{w}}}{v_{\mathrm{s}}^{3}}$ where $L$ is the observed luminosity at a particular epoch, and $\epsilon$ represents the efficiency of converting the shock's kinetic energy into visual light. $v_{\mathrm{w}}$ and $v_{\mathrm{s}}$ are the velocities of the pre-explosion stellar wind and the post-shock shell, respectively. We adopt a magnitude of $M_{\mathrm{v}}=-20.8 \pm 0.1$ on day +27 by interpolating the Swift magnitude provided in Table 2 and estimate the luminosity to be $L=\sim 6.0 \times 10^{42} \mathrm{erg} \mathrm{s}^{-1}$. No bolometric correction has been applied in this calculation. Assuming a constant value of $v_{\mathrm{w}}=$ $20 \mathrm{~km} \mathrm{~s}^{-1}$ for a red supergiant (RSG) wind and $v_{\mathrm{s}}=1862 \mathrm{~km}$ $\mathrm{s}^{-1}$ for the $\mathrm{SN}$ ejecta (see Section 3.2). For a value of $\epsilon=0.5$ (though, $\epsilon$ is optical depth dependent, Smith \& McCray 2007), $\dot{M}$ is estimated as $0.12 \mathrm{M}_{\odot} \mathrm{yr}^{-1}$. The typical mass-loss rates in RSGs, yellow hypergiants, and luminous blue variables (LBVs) are found to have a range of $10^{-6}-10^{-4}, 10^{-4}-10^{-3}$, and $0.1-10 \mathrm{M}_{\odot}$ $\mathrm{yr}^{-1}$, respectively (see Smith 2014). The mass-loss rate estimated for SN 2017hcc is closer to that seen in LBVs, indicating that its progenitor is likely to be an LBV star.

\subsection{Intrinsic polarization}

To derive the intrinsic polarization parameters of SN 2017hcc, ISP contribution must be subtracted from the observed polarization values. The intrinsic Stokes parameters were estimated by the relations: $Q_{\text {int }}=Q_{\text {obs }}-Q_{\text {ISP }}$ and $U_{\text {int }}=U_{\text {obs }}-U_{\text {ISP }}$. Here $Q_{\text {obs }}, U_{\text {obs }}$ and $Q_{\text {ISP }}, U_{\text {ISP }}$ represent the observed and ISP Stokes parameters, respectively. Therefore, at each epoch vectorial subtraction was performed and the resulted $Q_{\text {int }}$ and $U_{\text {int }}$ were converted back to $P_{\text {int }}$ and $\theta_{\text {int }}$ with the help of following equations:

$P_{\text {int }}=\sqrt{Q_{\text {int }}^{2}+U_{\text {int }}^{2}}$

$\theta_{\text {int }}=0.5 \times \arctan \left(\frac{U_{\text {int }}}{Q_{\text {int }}}\right)$

Both the observed and the intrinsic (ISP corrected) polarization parameters of SN 2017hcc are listed in Table 4 along with the spectropolarimetric observation of Mauerhan et al. (2017b).

The polarization in $V$ band at $\sim+2 \mathrm{~d}$ was estimated as $4.84 \pm 0.03$ percent by Mauerhan et al. (2017b), and a value of $1.36 \pm 0.10$ percent is estimated at $\sim+59 \mathrm{~d}$. The $R$-band polarization at $\sim+13$ and $\sim+56 \mathrm{~d}$ are estimated at $3.22 \pm 0.13$ and $1.35 \pm 0.20$ per cent, respectively. The position angle in both the bands is $\sim 100^{\circ}$ at all epochs. Accounting for the maximum ISP contribution (see Section 3.1), marginal changes are seen in the intrinsic polarization values. Here, a $V$-band polarization of 4.84 per cent $\left(95.0^{\circ}\right)$ and 1.34 per cent $\left(98.9^{\circ}\right)$ were estimated on the $\sim+2$ and $\sim+59 \mathrm{~d}$, respectively. Similarly, an $R$-band polarization of 3.22 percent $\left(94.1^{\circ}\right)$ and 1.33 percent $\left(99.5^{\circ}\right)$ were estimated on $\sim+13$ and $\sim+56 \mathrm{~d}$, respectively. This clearly indicates that the observed polarization values are intrinsic, and the degree of polarization decreased by $\sim 3.5$ percent in $V$ band, and by $\sim 2.3$ percent in the $R$ band within a period of $\sim 2$ months. No significant variation is observed in the polarization angle in both the bands during this period.

\subsection{Comparison of SN 2017hcc with other Type IIn events}

Only a handful of Type IIn SNe other than SN 2017hcc have been monitored polarimetrically close to the maximum. These are SN 1997eg (Hoffman et al. 2008), SN 1998S (Li et al. 1998), SN 2009ip (Mauerhan et al. 2014; Reilly et al. 2017), SN 2010j1 
Table 4. Observed and intrinsic polarization parameters of SN 2017hcc.

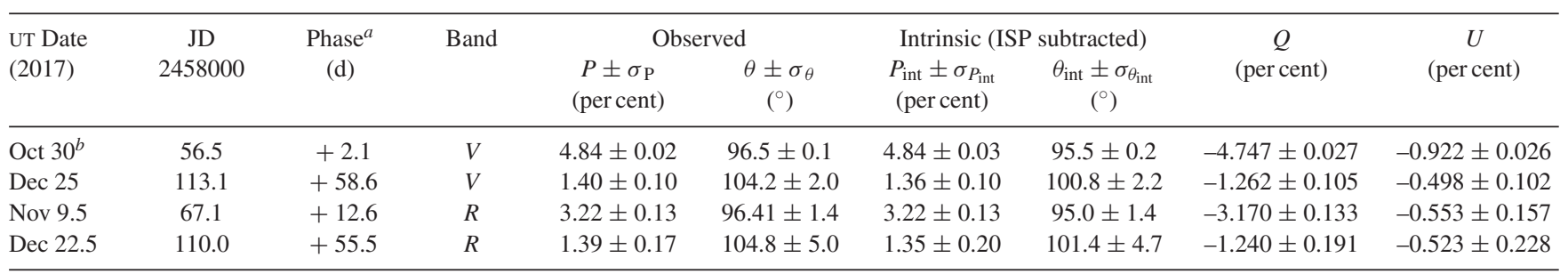

Notes. ${ }^{a}$ With reference to the maximum light JD 2458054.5.

${ }^{b}$ Observation of this epoch has been reported by Mauerhan et al. (2017b).

(Newton \& Puckett 2010), and SN 2012ab (Vinko et al. 2012). The evolution of polarization parameters of SN $2017 \mathrm{hcc}$ along with the above events is shown in Fig. 7. In general, the temporal evolution in the intrinsic polarization of the comparison sample (except SN 2009ip) display an increasing trend with no significant variation in their polarization angle. In the case of SN 1997eg, Hoffman et al. (2008) discussed the polarization properties of SN 1997eg in the context of three ISP values. Their preferred ISP subtracted polarization exhibited an increasing trend whereas the other two ISP values resulted in decreasing trend of the intrinsic polarization (see fig. 11 in Hoffman et al. 2008).

The peculiar SN 2009ip (2012b outburst, Mauerhan et al. 2013; Prieto, Brimacombe, Drake \& Howerton 2013; Smith, Mauerhan \& Prieto 2014) displayed a high degree of polarization $\left(P_{\mathrm{V}} \sim 1.7\right.$ per cent $)$ at maximum $\left(M_{\mathrm{V}} \sim-18.5 \mathrm{mag}\right)$ and followed a decreasing trend similar to the decay in its light curve, until day $\sim 70$. This behaviour is indicative of a correlation between the intrinsic polarization and photometric luminosity. A similar trend is seen in $\mathrm{SN} 2017 \mathrm{hcc}$, with a higher intrinsic polarization $\left(P_{\mathrm{V}} \sim 4.84\right.$ percent $)$, and a brighter maximum $\left(M_{\mathrm{V}} \sim-20.7 \mathrm{mag}\right)$. Further, there is almost no variation seen in the polarization angle in the case of SN $2017 \mathrm{hcc}$, whereas, a substantial variation $\left(\sim 30^{\circ}\right.$ was seen in the case of SN 2009ip (Mauerhan et al. 2014; Reilly et al. 2017).

The emergence of polarization among Type IIn SNe is generally attributed to either electron scattering in an asymmetric photosphere, or asymmetries in the interaction region. In several studies, departure of the SN ejecta from spherical symmetry is a favoured explanation. This line of reasoning makes it rather straightforward to explain the temporal increase of intrinsic polarization as the core becomes progressively exposed. This also suggests that the inner layers of the ejecta are quite aspherical, indicating an aspherical explosion (see Wang et al. 1996; Tran et al. 1997; Kawabata et al. 2002; Leonard et al. 2006; Maund et al. 2007a, b; Chornock et al. 2010; Kumar et al. 2014, 2016; Mauerhan et al. 2017a, and references therein).

The maximum light in Type IIn SNe is primarily powered by an interaction of the ejecta with a massive CSM, implying that the major source of intrinsic polarization at maximum is due to this interaction, particularly with an asymmetric CSM. The luminosity from the interaction becomes progressively weaker as the CSM around the SN evolves into an optically thin state. The asymmetry in the SN photosphere (resulting from an aspherical explosion) can also complement the polarization signal arising from the CSM. The percentage contribution from the photosphere increases temporally and plays a dominant role during the later phases as the luminosity from interaction dies off slowly. Overall, the relative contribution

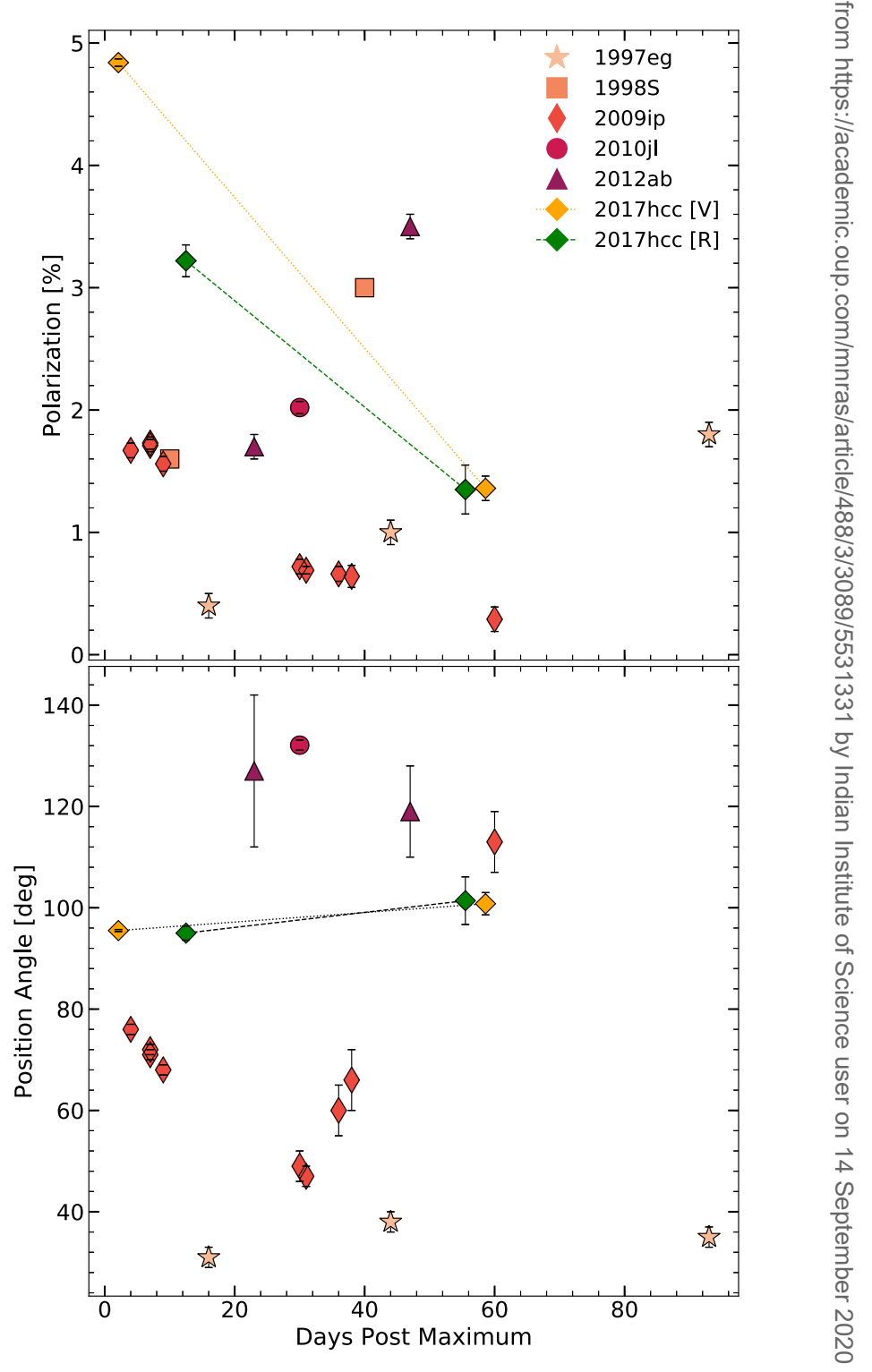

Figure 7. Polarization evolution of SN 2017hec in $V$ and $R$ bands are shown with different symbols and connected with lines for clarity (top panel: intrinsic polarization values and bottom panel: position angle). Polarization inferred from SN 2012ab (Bilinski et al. 2018), SN 2010j1 (Patat et al. 2011), SN 2009ip (Mauerhan et al. 2014), SN 1998S (Wang et al. 2001), and SN 1997eg (Hoffman et al. 2008) have been plotted for comparison. 
to the net luminosity from the photosphere and the CSM interaction decides the strength of net intrinsic polarization.

The high degree of intrinsic polarization at initial phases displayed by SN 2017hcc is an exceptional example among CCSNe. A significant decline of $\sim 3.5$ per cent in the intrinsic polarization was seen within a period of $\sim 2$ months. During the late phase ( $\sim 2$ months from maximum) polarization values are wavelength independent (cf. $\sim 1.4$ percent polarization in $V$ and $R$ bands). The decline in polarization seen in SN 2017hcc is very similar to the early decline observed in SN 2009ip (Mauerhan et al. 2014), and explained as due to interaction with an aspherical CSM. As discussed in Section 3.3, the progenitor of SN 2017hcc is likely an LBV star. These type of stars undergo episodic mass loss or giant eruption immediately before the explosion leading to the formation of circumstellar shells and/or equatorial disks/rings around them (Smith 2017). These structures distort a spherically symmetric $\mathrm{SN}$ photosphere upon interaction, enhancing the advancing polarization signal. While a detailed modelling is beyond the scope of this work, it appears that the strong polarization seen in SN 2017hcc could be a possible outcome of an interaction of the SN ejecta with an highly aspheric CSM (Mauerhan et al. 2017b).

Additionally, one cannot rule out other possibilities, such as net reduction in the number density of the scatterers (such as electrons and dust grains) as a function of time in a non-spherical ejecta. This is expected because the cooling in the SN ejecta as a function of time (cf. star symbols in Fig. 6) would result in a corresponding decay in the electron number density. Furthermore, the continuous expansion of SN ejecta as a function of time (cf. blue filled circles in Fig. 6) may also cause a net reduction in the clumpiness (if any) leading to a decrease in the net polarization.

\section{SUMMARY}

We have presented imaging (Swift UVOT), spectroscopy and polarimetry of the luminous Type IIn SN 2017hcc and compared the results with similar events. The light curve evolution was very slow during post-maximum phase $\left(\sim 0.2 \mathrm{mag} 100 \mathrm{~d}^{-1}\right.$ in $b$ band during $\sim 0-45 \mathrm{~d}$ ). The $u v w 1, u v w 2$, and $u v m 2$ band light curves evolved with similar rate however, the evolution was almost flat in $b$ and $v$ bands. The broad-band polarization characteristics were examined following robustly determined ISP contribution, which is found to be 0.17 percent and $140^{\circ}$ (mainly arising from the Galactic dust and assuming a mean polarization efficiency). The intrinsic degree of polarization (in $V$ band) near the maximum light was $\sim 4.84$ percent which reduced to $\sim 1.36$ percent after $\sim 2$ months. Similarly in $R$ band also, the intrinsic polarization values are high i.e. 3.22 percent $(\sim+13$ d) and 1.35 percent $(\sim+56 \mathrm{~d})$. Such large amount of polarization is unusual for an $\mathrm{SN}$. Interestingly, the intrinsic polarization angle was found to remain constant around $\sim 100^{\circ}$ at all epochs. There is no significant change in the intrinsic polarization parameters even if maximum polarization efficiency relation is considered towards the $\mathrm{SN}$ direction.

We note that SN 2017hcc is not only peculiar in terms of polarization inferred but also belongs to the group of bright Type IIn events $\left(M_{\mathrm{V}}=-20.7 \mathrm{mag}\right.$, Prieto et al. 2017). Its peak luminosity is equivalent to SN $2006 \mathrm{tf}\left(M_{\mathrm{V}}=-20.8 \mathrm{mag}\right.$, Smith et al. 2008). The $\sim 27$ d post-maximum spectrum of SN 2017 hcc exhibited prominent narrow lines of $\mathrm{H}$ and $\mathrm{He}$ as typically seen in Type IIn SNe but the spectrum is bluer relative to SN 2010jl and SN 2012ab. The evolution of blackbody temperature and radius signifies a hotter and optically thick environment of SN 2017hcc than those of typical Type IIn SNe. The NaI D lines are not visible in our spectrum, indicating a negligible host extinction. The mass-loss rate of SN 2017 hcc is estimated to be $0.12 \mathrm{M}_{\odot} \mathrm{yr}^{-1}$ based on a simple CSM-ejecta interaction model which suggests an LBV progenitor for this event.

The high degree of polarization values found in SN 2017hcc support the fact that events belonging to this class are in general significantly polarized in comparison to other CCSNe and thermonuclear SNe (Type Ia, Wang \& Wheeler 2008; Patat 2017). A disc-like or toroidal CSM around the ejecta seems to be a common characteristic among Type IIn SNe that induces polarization signal. As described in Section 3.4, diversity exists in the polarization properties of Type IIn SNe. This indicates variation in the electronscattering atmosphere which is regulated by complexity of the SN ejecta and/or CSM composition. A cumulative effect of the highly aspherical CSM and the ejecta density profile may have produced such a strong polarization in SN 2017hcc without significant change in the overall geometry (Kasen et al. 2003; Kasen, Thomas \& Nugent 2006; Hole, Kasen \& Nordsieck 2010; Tanaka et al. 2017). Better temporal coverage in polarimetric monitoring could reveal a wealth of crucial information including the CSM/ejecta geometry, clumpy structure and underlying progenitor nature etc. In this context, the upcoming large aperture observing facilities shall be extremely useful.

\section{ACKNOWLEDGEMENTS}

We thank the referee for his/her several useful comments that have significantly improved the manuscript. We also thank Masaomi Tanaka for useful discussions on various polarimetric aspects and Ashish Raj who kindly agreed to obtain HFOSC spectra during his observations. BK acknowledges the Science and Engineering Research Board (SERB) under the Department of Science and Technology, Govt. of India, for financial assistance in the form of National Post-Doctoral Fellowship (ref. no. PDF/2016/001563). CE acknowledges (i) The National Key R\&D Program of China grant no. 2017YFA0402600 and (ii) The International Partnership Program of Chinese Academy of Sciences grant no. 114A11KYSB20160008. BK, DKS, and GCA also acknowledge BRICS grant DST/IMRCD/BRICS/PilotCall1/MuMeSTU/2017(G) for the present work. DKS and GCA also acknowledge DST/JSPS grant, DST/INT/JSPS/P/281/2018. SBP acknowledges BRICS grant DST/IMRCD/BRICS/Pilotcall/ProFCheap/2017(G) for this work.

We thank the staff of IAO, Hanle and CREST, Hosakote that made HFOSC observations possible. The facilities at IAO and CREST are operated by the Indian Institute of Astrophysics, Bangalore. This work has made use of data from the European Space Agency (ESA) mission Gaia (https://www.cosmos.esa.int/gaia), processed by the Gaia Data Processing and Analysis Consortium (DPAC, https: //www.cosmos.esa.int/web/gaia/dpac/consortium). Funding for the DPAC has been provided by national institutions, in particular the institutions participating in the Gaia Multilateral Agreement. This research has made use of the SIMBAD data base, operated at CDS, Strasbourg, France. This research made use of REDPIPE ${ }^{6}$, an assemblage of data reduction and analysis scripts written by AS.

\footnotetext{
${ }^{6}$ https://github.com/sPaMFouR/RedPipe
} 


\section{REFERENCES}

Akitaya H. et al., 2014, in Ramsay S. K., McLean I. S., Takami H., eds, SPIE Conf. Ser. Vol. 9147, Ground-based and Airborne Instrumentation for Astronomy V. SPIE, Bellingham,p. 914740

Bilinski C. et al., 2018, MNRAS, 475, 1104

Bose S., Kumar B., Misra K., Matsumoto K., Kumar B., Singh M., Fukushima D., Kawabata M., 2016, MNRAS, 455, 2712

Branch D., Wheeler J. C., 2017, Supernova Explosions. Springer-Verlag GmbH, Germany

Chandra P., Fransson C., Chevalier R. A., 2017, Astron. Telegram, 10936, 1

Chevalier R. A., Fransson C., 1994, ApJ, 420, 268

Chornock R., Filippenko A. V., Li W., Silverman J. M., 2010, ApJ, 713, 1363

Chugai N. N., 2001, MNRAS, 326, 1448

Chugai N. N., Danziger I. J., 1994, MNRAS, 268, 173

Dastidar R. et al., 2019, MNRAS, 486, 2850

Das H. S., Medhi B. J., Wolf S., Bertrang G., Deb Roy P., Chakraborty A., 2013, MNRAS, 436, 3500

Dwarkadas V. V., 2011, MNRAS, 412, 1639

Eswaraiah C. et al., 2019, ApJ, 875, 64

Eswaraiah C., Pandey A. K., Maheswar G., Chen W. P., Ojha D. K., Chandola H. C., 2012, MNRAS, 419, 2587

Eswaraiah C., Maheswar G., Pandey A. K., Jose J., Ramaprakash A. N., Bhatt H. C., 2013, A\&A, 556, A65

Fassia A. et al., 2001, MNRAS, 325, 907

Filippenko A. V., 1997, ARA\&A, 35, 309

Fox O. D., Filippenko A. V., Skrutskie M. F., Silverman J. M., Ganeshalingam M., Cenko S. B., Clubb K. I., 2013, AJ, 146, 2

Fransson C. et al., 2002, ApJ, 572, 350

Gaia Collaboration et al., 2018, A\&A, 616, A1

Gal-Yam A. et al., 2007, ApJ, 656, 372

Gangopadhyay A. et al., 2019, MNRAS, in press

Gavrilchenko T., Klein C. R., Bloom J. S., Richards J. W., 2014, MNRAS, 441,715

Green G. M. et al., 2018, MNRAS, 478, 651

Habergham S. M., Anderson J. P., James P. A., Lyman J. D., 2014, MNRAS, 441,2230

Heiles C., 2000, AJ, 119, 923

Hoffman J. L., Leonard D. C., Chornock R., Filippenko A. V., Barth A. J., Matheson T., 2008, ApJ, 688, 1186

Hoflich P., 1991, A\&A, 246, 481

Hole K. T., Kasen D., Nordsieck K. H., 2010, ApJ, 720, 1500

Kasen D. et al., 2003, ApJ, 593, 788

Kasen D., Thomas R. C., Nugent P., 2006, ApJ, 651, 366

Kawabata K. S. et al., 2002, ApJ, 580, L39

Kiewe M. et al., 2012, ApJ, 744, 10

Kumar B., Pandey S. B., Eswaraiah C., Gorosabel J., 2014, MNRAS, 442, 2

Kumar B., Pandey S. B., Eswaraiah C., Kawabata K. S., 2016, MNRAS, 456,3157

Kumar B., Singh A., Srivastav S., Sahu D. K., Anupama G. C., 2018, MNRAS, 473, 3776

Lee C. D., Eswaraiah C., Chen W. P., Pandey A. K., 2018, AJ, 156, 115

Leonard D. C. et al., 2006, Nature, 440, 505

Leonard D. C., Filippenko A. V., 2005, in Turatto M., Benetti S., Zampieri L., Shea W., eds, ASP Conf. Ser. Vol. 342, 1604-2004: Supernovae as Cosmological Lighthouses. Astron. Soc. Pac., San Francisco,p. 330

Leonard D. C., Filippenko A. V., Barth A. J., Matheson T., 2000, ApJ, 536, 239

Li W., Chornock R., Leaman J., Filippenko A. V., Poznanski D., Wang X., Ganeshalingam M., Mannucci F., 2011, MNRAS, 412, 1473

Li W.-D., Li C., Filippenko A. V., Moran E. C., 1998, IAU Circ., 6829, 1

Mauerhan J. et al., 2014, MNRAS, 442, 1166

Mauerhan J. C. et al., 2013, MNRAS, 430, 1801

Mauerhan J. C. et al., 2017a, ApJ, 834, 118

Mauerhan J. C., Filippenko A. V., Brink T. G., Zheng W., 2017b, Astron. Telegram, 10911, 1
Maund J. R., Wheeler J. C., Patat F., Baade D., Wang L., Höflich P., 2007a, MNRAS, 381, 201

Maund J. R., Wheeler J. C., Patat F., Wang L., Baade D., Höflich P. A., 2007b, ApJ, 671, 1944

Medhi B. J., Maheswar G., Brijesh K., Pandey J. C., Kumar T. S., Sagar R., 2007, MNRAS, 378, 881

Moriya T. J., Tominaga N., Langer N., Nomoto K., Blinnikov S. I., Sorokina E. I., 2014, A\&A, 569, A57

Nayana A. J., Chandra P., 2017, Astron. Telegram, 11015, 1

Neha S., Maheswar G., Soam A., Lee C. W., Tej A., 2016, A\&A, 588, A45

Newton J., Puckett T., 2010, Central Bureau Electronic Telegrams, 2532, 1

Ofek E. O. et al., 2014, ApJ, 788, 154

Pandey J. C., Medhi B. J., Sagar R., Pandey A. K., 2009, MNRAS, 396, 1004

Pastorello A. et al., 2013, ApJ, 767, 1

Patat F., 2017, Introduction to Supernova Polarimetry. Handbook of Supernovae. Springer, Cham. p. 1017

Patat F., Taubenberger S., Benetti S., Pastorello A., Harutyunyan A., 2011, A\&A, 527, L6

Patel M. K., Pandey J. C., Savanov I. S., Prasad V., Srivastava D. C., 2013, MNRAS, 430, 2154

Poole T. S. et al., 2008, MNRAS, 383, 627

Poznanski D., Prochaska J. X., Bloom J. S., 2012, MNRAS, 426, 1465

Prieto J. L. et al., 2007, preprint(arXiv:0706.4088)

Prieto J. L. et al., 2017, Res. Notes AAS, 1, 28

Prieto J. L., Brimacombe J., Drake A. J., Howerton S., 2013, ApJ, 763, L27

Rakshit S., Stalin C. S., Muneer S., Neha S., Paliya V. S., 2017, ApJ, 835, 275

Ramaprakash A. N., Gupta R., Sen A. K., Tandon S. N., 1998, A\&AS, 128 , 369

Rautela B. S., Joshi G. C., Pandey J. C., 2004, Bull. Astron. Soc. India, 32, 159

Reilly E., Maund J. R., Baade D., Wheeler J. C., Höflich P., Spyromilio J., Patat F., Wang L., 2017, MNRAS, 470, 1491

Sahu D. K., Anupama G. C., Chakradhari N. K., Srivastav S., Tanaka M., Maeda K., Nomoto K., 2018, MNRAS, 475, 2591

Schlafly E. F., Finkbeiner D. P., 2011, ApJ, 737, 103

Schlegel E. M., 1990, MNRAS, 244, 269

Schmidt G. D., Elston R., Lupie O. L., 1992, AJ, 104, 1563

Serkowski K., Mathewson D. S., Ford V. L., 1975, ApJ, 196, 261

Shapiro P. R., Sutherland P. G., 1982, ApJ, 263, 902

Singh A., Srivastav S., Kumar B., Anupama G. C., Sahu D. K., 2018, MNRAS, 480, 2475

Singh A., Kumar B., Moriya T. J., Anupama G. C., Sahu D. K., Brown P. J., Andrews J. E., Smith N., 2019, ApJ, preprint (arXiv:1907.03159)

Smith N. et al., 2007, ApJ, 666, 1116

Smith N., 2014, ARA\&A, 52, 487

Smith N., 2017, Interacting Supernovae: Types IIn and Ibn. Handbook of Supernovae. Springer, Cham., p. 403

Smith N., McCray R., 2007, ApJ, 671, L17

Smith N., Chornock R., Li W., Ganeshalingam M., Silverman J. M., Foley R. J., Filippenko A. V., Barth A. J., 2008, ApJ, 686, 467

Smith N., Chornock R., Silverman J. M., Filippenko A. V., Foley R. J., 2010, ApJ, 709, 856

Smith N., Mauerhan J. C., Prieto J. L., 2014, MNRAS, 438, 1191

Soam A., Maheswar G., Lee C. W., Dib S., Bhatt H. C., Tamura M., Kim G., 2015, A\&A, 573, A34

Srivastav S., Ninan J. P., Kumar B., Anupama G. C., Sahu D. K., Ojha D. K., Prabhu T. P., 2016, MNRAS, 457, 1000

Stritzinger M. D. et al., 2018, A\&A, 609, A135

Taddia F. et al., 2013, A\&A, 555, A10

Taddia F. et al., 2015, A\&A, 580, A131

Tanaka M., Maeda K., Mazzali P. A., Kawabata K. S., Nomoto K., 2017, ApJ, 837, 105

Tonry J. L., 2011, PASP, 123, 58

Tran H. D., 1995, ApJ, 440, 565

Tran H. D., Filippenko A. V., Schmidt G. D., Bjorkman K. S., Jannuzi B. T., Smith P. S., 1997, PASP, 109, 489 
Turatto M., Benetti S., Cappellaro E., 2003, in Hillebrandt W., Leibundgut B., eds, From Twilight to Highlight: The Physics of Supernovae. Springer-Verlag, Berlin, p. 200

Vinko J. et al., 2012, Central Bureau Electronic Telegrams, 3022

Wang L., Wheeler J. C., 2008, ARA\&A, 46, 433

Wang L., Wheeler J. C., Li Z., Clocchiatti A., 1996, ApJ, 467, 435

Wang L., Howell D. A., Höflich P., Wheeler J. C., 2001, ApJ, 550, 1030
Wang J.-W., Lai S.-P., Eswaraiah C., Clemens D. P., Chen W.-P., Pandey A. K., 2017, ApJ, 849, 157

Whittet D. C. B., van Breda I. G., 1978, A\&A, 66, 57

Zhang T. et al., 2012, AJ, 144, 131

This paper has been typeset from a $\mathrm{T}_{\mathrm{E}} \mathrm{X} / \mathrm{L} \mathrm{A} \mathrm{E} \mathrm{X}$ file prepared by the author. 